\title{
Hiperinsulinismo endógeno diagnosticado como epilepsia
}

\author{
Alejandro Pinzón Tovar ${ }^{1}$, Samuel Yucumá Gutiérrez²
}

\author{
${ }^{1}$ Médico Internista y Endocrinólogo, docente catedrático \\ Universidad Surcolombiana, director científico ENDHO Colombia. \\ El autor ha sido conferencista para Sanofi; Novartis, Novo nordisk \\ $y$ Glaxo en temas de diabetes y trastornos tiroideos. \\ ${ }^{2}$ Médico Internista, Universidad Surcolombiana. \\ Correo electrónico: mdsamuel@hotmail.com \\ Conflicto de interés: El presente artículo no contó con fuen- \\ tes de financiación externa. \\ Fecha de recepción: $17 / 11 / 2015$ \\ Fecha de aceptación: 2/02/2016
}

\section{Resumen}

$\mathrm{E}$ l insulinoma es la neoplasia endocrina más común del páncreas, $90 \%$ de ellos son benignos, su incidencia es de 4 por 1 millón de personas al año y representa el 1\% al $2 \%$ de todas las neoplasias pancreáticas. Los insulinomas son la causa más común de hipoglucemia relacionada con hiperinsulinismo endógeno. La naturaleza episódica del ataque hipoglucémico se debe a la secreción intermitente de insulina por el tumor. En adultos, la confirmación biológica y bioquímica de la patología al igual que la búsqueda de su localización se debe realizar luego de la resolución de los episodios de hipoglucemia. El diagnóstico de insulinoma puede ser un reto, patologías psiquiátricas o neurológicas son diagnosticadas de manera errónea antes de llegar a la identificación del tumor en células de los islotes pancreáticos. La resección quirúrgica es la modalidad de tratamiento primario, con la que se resuelve la sintomatología cuando se logra la completa resección del tumor.

Palabras clave: páncreas; hiperinsulinismo endógeno; Insulinoma; hipoglucemia; epilepsia; tumor pancreático neuroendocrino.

\section{Abstract}

The insulinoma is the most common pancreatic endocrine neoplasia, 90\% of them are benign, the incidence is 4 per 1 million people a year and accounts for $1 \%$ to $2 \%$ of all pancreatic neoplasms. Insulinomas are the most common cause of hypoglycemia associated with endogenous hyperinsulinism. The episodic nature of hypoglycemic attack is due to the intermittent insulin secretion by the tumor. In adults, biological and biochemical confirmation of pathology like finding your location should be performed after resolution of hypoglycemic episodes. The diagnosis of insulinoma can be challenging, psychiatric or neurological disorders are diagnosed incorrectly before reaching identifying tumor cells of pancreatic islets. Surgical resection is the primary treatment modality with which the symptoms resolved when complete resection of the tumor is achieved.

Key words: pancreas; endogenous hyperinsulinism; insulinoma; hypoglycemia; epilepsy; neuroendocrine pancreatic tumor.

\section{Introducción}

Las células de los islotes pancreáticos fueron descritas por Paul Langerhans en 1869; Harris en 1923, sugirió la posibilidad clínica de hiperinsulinismo; sin embargo, fue tres años más tarde cuando Wilder estableció por primera vez la asociación entre dicha patología y el tumor de las células de los islotes pancreáticos mediante la intervención quirúrgica realizada a un paciente con hipoglucemia, donde encontró carcinoma de células de los islotes pancreáticos con metástasis hepáticas ${ }^{(1)}$.

La base para el diagnóstico del insulinoma fue dada en 1935 por Whipple, quien observó que los pacientes con hipoglucemia y niveles de glucosa menores de $50 \mathrm{mg} / \mathrm{dl}$ resolvían sus síntomas con la administración de glucosa, estableciendo de esta manera la "tríada de Whipple", la cual se utiliza actualmente en el diagnóstico de hipoglucemia en el paciente no diabético ${ }^{(1,2,3,4)}$.

El insulinoma es la neoplasia endocrina más común del páncreas, se caracteriza por la presencia de tumores que segregan insulina y que causan estados de hipoglucemia; su incidencia es de 4 por 1 millón de personas al año ${ }^{(1,2)}$. Pueden ocurrir a cualquier edad, con una distribución equitativa entre los géneros, y por fortuna $90 \%$ de los casos son benignos ${ }^{(1,2,5,6,7)}$.

En los adultos con síntomas neuroglucopénicos y niveles bajos de glucosa (menores a $55 \mathrm{mg} / \mathrm{dl}$ ), el estándar de oro para el diagnóstico bioquímico sigue siendo la medición de la glucosa en plasma, la determinación de insulina, péptido $\mathrm{C}$ y proinsulina durante el ayuno, descartando la presencia de sulfonilureas en plasma u orina. Estas pruebas pueden detectar hasta el 99\% de los casos de hiperinsulinismo endógeno ${ }^{(2)}$.

Tras su confirmación bioquímica, la localización preoperatoria de la neoplasia se realiza mediante tomografía computarizada (TC), resonancia magnética (RM), ultrasonografía endoscópica (USE), prueba de estimulación arterial selectiva con 
calcio, muestreo venoso portal transhepático y/o angiografía. En la actualidad, el tratamiento primario para la resolución de los síntomas consiste en la resección quirúrgica en el caso de los insulinomas ${ }^{(1,2,7,8)}$.

La epilepsia es un trastorno común y su diagnóstico en muchos casos sigue basándose en la presentación clínica. Este enfoque diagnóstico puede conducir al inicio de fármacos antiepilépticos sin un diagnóstico claro. Las crisis epilépticas pueden ser imitadas por muchas condiciones, incluyendo la hipoglucemia y otros trastornos metabólicos. Se presenta el caso de un paciente con insulinoma que inicialmente fue diagnosticado como epilepsia y tratado con anticonvulsivantes durante un año antes de realizar el diagnóstico correcto.

\section{Presentación del caso}

Paciente de 51 años, de sexo masculino, procedente de área rural del Caquetá, con cuadro clínico de un año de evolución consistente en mareos y episodios convulsivos con pérdida de la conciencia; sintomatología que fue interpretada como epilepsia sintomática, para la cual recibió múltiples esquemas de tratamiento anticonvulsivante. Durante su última hospitalización, se documentó un valor de glucemia de $23 \mathrm{mg} / \mathrm{dl}$. Se logró establecer una clara relación entre los niveles bajos de glucosa sanguínea y su sintomatología, que requirió infusiones altas de dextrosa (entre 8 y 10 g/hora) para mantener en el límite inferior de la normalidad sus glucometrías. No existen antecedentes de importancia y no hay consumo de sulfonilureas. Al examen físico, el paciente se encuentra hemodinámicamente estable sin aparente déficit neurológico.

Durante un episodio de hipoglucemia se realiza medición de insulina basal con reporte de $17 \mathrm{mcU} / \mathrm{ml}$; péptido $\mathrm{C}$ en 2,57 $\mathrm{ng} / \mathrm{ml}$; y se corrobora ausencia de sulfonilureas en plasma; en TAC de abdomen se evidencia masa en cabeza de páncreas (figura 1) y con ultrasonografía endoscópica (USE) se reporta a nivel de la cabeza del páncreas, una lesión hipoecoica homogénea de bordes irregulares de 1,8 x 1,3 cm. (figura 2). Se realizó aspirado con aguja fina (ACAF) que muestra hallazgos sugestivos de neoplasia de células insulares por lo que se interconsulta a cirugía gastrointestinal, donde deciden programar pacreatoduodenectomía. Durante la intervención se encuentra lesión tumoral encapsulada a nivel del proceso uncinado del páncreas, sin hallar adenopatías tumorales; el hígado y el resto de cavidad abdominal es de apariencia normal; motivo por el cual se realiza resección de la masa pancreática con normalización posterior e inmediata de los niveles de glucosa sanguínea, tolerando la reducción de la dextrosa endovenosa. El reporte de patología es de un tumor endocrino bien diferenciado en contacto con el borde de resección, confirmando el diagnóstico de hipoglucemia hiperinsulinémica secundaria a insulinoma resecado. Durante su posoperatorio, el paciente tolera adecuadamente la vía oral y no presenta nuevos episo- dios de hipoglucemias, y en sus seguimientos por endocrinología ha permanecido asintomático durante los tres primeros años de control.

\section{Discusión}

El insulinoma es un tumor neuroendocrino poco frecuente. Es la neoplasia endocrina más común del páncreas y representa del $1 \%$ al $2 \%$ de todas las neoplasias pancreáticas, puede presentarse a cualquier edad, con una distribución equitativa entre los géneros. El $90 \%$ de los insulinomas son

\section{Figura 1. TAC abdomen contrastado}

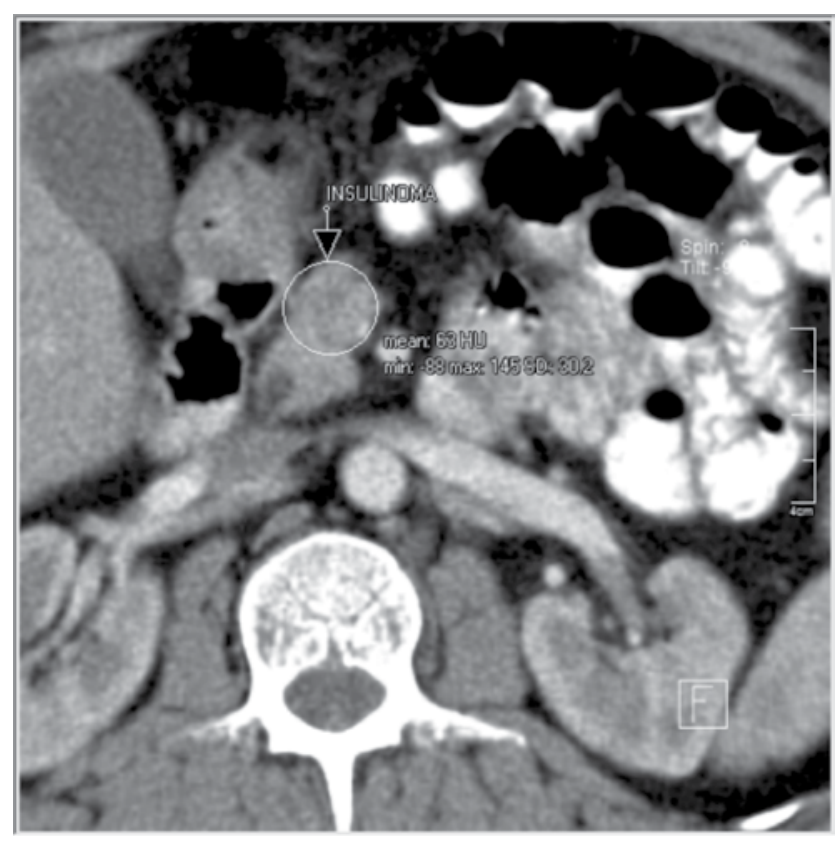

Lesión redondeada, hipodensa, con realce leve con el medio de contraste a nivel del proceso uncinado.

Figura 2. Ultrasonografía endoscópica

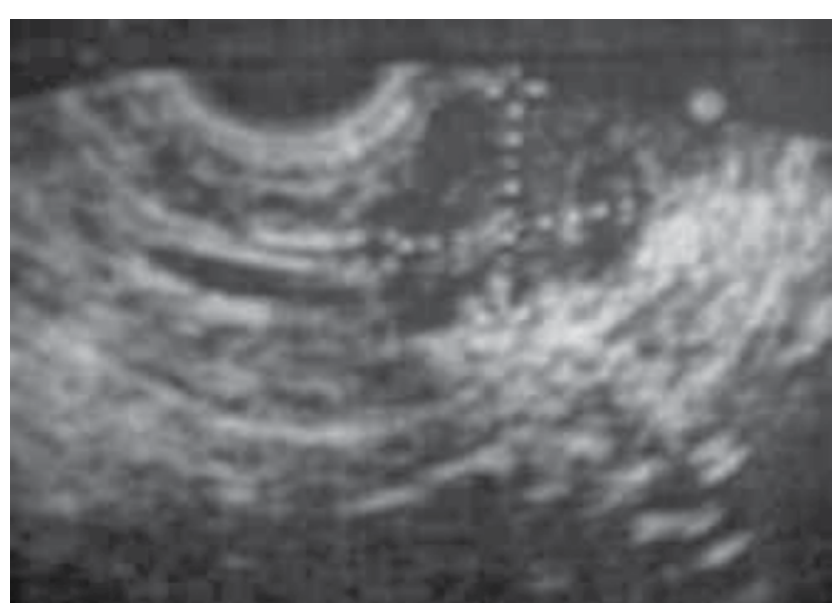

En cabeza de páncreas hacia el proceso uncinado se observa lesión hipoecoica de bordes irregulares, de aproximadamente $1,8 \times 1,3 \mathrm{~cm}$. 
benignos, más del $90 \%$ se origina en sitios intrapancreáticos distribuidos por igual en cabeza, cuerpo y cola. Los insulinomas extrapancréaticos que causan hipoglucemia son muy poco frecuentes $(<2 \%)$ y se localizan más comúnmente en la pared duodenal ${ }^{(1,2,9)}$.

Algunos estudios han demostrado que esta patología puede ocurrir de manera esporádica o estar asociada a una neoplasia endocrina múltiple tipo 1 (MEN-1), que se presenta por un trastorno autosómico dominante producido como resultado de la inactivación de las mutaciones del gen MEN1 localizado en el cromosoma 11, y se caracteriza por presentar hiperparatiroidismo primario, adenomas de la hipófisis anterior y la presencia de tumores del páncreas endocrino y/o del duodeno ${ }^{(10)}$

El insulinoma afecta aproximadamente al 10\% de los MEN1 , tienden a ser multifocales y se pueden localizar en todo el páncreas, en contraste con los insulinomas esporádicos, que típicamente se presentan en forma de lesiones solitarias, benignas y encapsuladas ${ }^{(10)}$. Los tumores de células de los islotes funcionales más comunes son gastrinomas e insulinomas. Los insulinomas por lo general son pequeños al momento del diagnóstico, el 90\% de los tumores tienen un diámetro menor a $2 \mathrm{~cm}^{(11,12)}$.

Este tipo de neoplasia es la causa más común de hipoglucemia relacionada con hiperinsulinismo endógeno y se produce por la secreción intermitente de la insulina por el tumor. Los síntomas autonómicos comunes de un insulinoma incluyen los neuroglucopénicos; como somnolencia, trastornos visuales, confusión, letargo, debilidad, comportamiento anormal, convulsiones, pérdida del conocimiento o coma. Además, se produce sobrestimulación simpática debido a la liberación de cotecolaminas, dando como resultado sudoración, ansiedad y palpitaciones. Estos síntomas se precipitan típicamente por el ayuno o el ejercicio, pero también pueden ocurrir después de las comidas, o tener relación con la ingesta de alimentos ${ }^{(1,2,3,4)}$.

Uno de los diagnósticos erróneos en pacientes que cumplen con la sintomatología descrita es el de epilepsia, como lo ocurrido con el paciente objeto de nuestra investigación. En un estudio retrospectivo realizado por Dizon et al. en 1999, el $64 \%$ de 58 pacientes con diagnóstico confirmado de tumor de células de los islotes pancreáticos tuvieron un primer diagnóstico de trastorno neurológico, incluyendo 39\% con epilepsia, antes de recibir un diagnóstico de insulinoma ${ }^{6,13,14)}$.

En la actualidad el diagnóstico de epilepsia continúa basándose en gran medida en su presentación clínica, los registros electrofisiológicos se utilizan para evaluar las anomalías interictales y para registrar y caracterizar las convulsiones. Las imágenes cerebrales, son útiles para efectos etiológicos con el fin de identificar anomalías estructurales cuando están presentes. Sin embargo, este enfoque diagnóstico puede conducir a iniciar el tratamiento con uno o más fármacos antiepilépticos, sin un diagnóstico claro de la enfermedad ${ }^{(6,15,16)}$. En los centros de referencia de epilepsia, la tasa de diagnóstico erróneo está entre el 25\% y $30 \%$ para los pacientes que se someten a vigilancia por video-EEG prolongada ${ }^{(14)}$.

El insulinoma muchas veces puede presentar síntomas neuroglucopénicos variados como amnesia, dificultad de concentración, ansiedad, síntomas obsesivos-compulsivos, histrionismo, depresión, psicosis con alucinaciones, ideas de suicidio, delirium, estupor, coma y demencia, además de cefalea, diplopía, convulsiones, hemiplejía, afasia, arreflexia y acinesia. Por lo anterior, un porcentaje elevado de pacientes han sido diagnosticados previamente con diversas enfermedades psiquiátricas y neurológicas, entre ellas trastorno de pánico, trastorno de personalidad, demencia, psicosis, manía, depresión, epilepsia etc. ${ }^{(21,22)}$.

Tras la confirmación bioquímica durante el episodio de hipoglucemia de insulina sérica mayor a $3 \mathrm{mcU} / \mathrm{ml}$, péptido C mayor a $0,2 \mathrm{nmol} / \mathrm{L}(0,6 \mathrm{ng} / \mathrm{ml})$ y proinsulina mayor a $5 \mathrm{pmol} / \mathrm{L}$, en ausencia de cualquier agente hipoglucemiante circulante y anticuerpos para la insulina negativos, se realiza el diagnóstico de hiperinsulinismo endógeno ${ }^{(3)}$. En adultos se debe sospechar la presencia de un insulinoma, la localización del tumor se realiza por medio de estudios imaginológicos, los cuales también sirven para evaluar la presencia de enfermedad metastásica y para definir el tipo de técnica quirúrgica a desarrollar. Los estudios utilizados son: ultrasonografía endoscópica (USE), tomografía computarizada (TC), resonancia magnética (RM), prueba de estimulación arterial selectiva con calcio, muestreo venoso portal transhepático y/o angiografía. Sin embargo, a pesar de estos procedimientos, en $10 \%$ a $27 \%$ de los casos no se logra la localización del tumor ${ }^{(1,2,11,12)}$.

En cuanto a la ultrasonografía endoscópica (USE), su tasa de éxito varía ampliamente, entre 9\% y 66\%. Aunque la USE es un procedimiento altamente fiable para la localización preoperatoria de los insulinomas, existen varios problemas asociados; en primer lugar, depende en gran medida de la experiencia del examinador; en segundo lugar, algunos insulinomas no se logran visualizar por ser isoecoicos; y en tercer lugar, la sensibilidad de la USE para insulinomas depende de la ubicación y del tamaño del tumor; siendo mayor para los tumores ubicados en la cabeza del páncreas y más baja para aquellos de la cola del páncreas o los extrapancreáticos. Si el tumor se ha localizado, la aspiración con aguja fina (ACAF) puede permitir un diagnóstico prequirúrgico(2).

La TC helicoidal localiza entre 50\% y $80 \%$ de los tumores. Sin embargo, en un reciente informe se ha visualizado con esta técnica el 94,4\% de los insulinomas, es por ello que actualmente es aceptada como la imagen de primera línea para el diagnóstico preoperatorio. Entre el $40 \%$ y el $70 \%$ de los insulinomas son detectados por RM y cerca de la mitad por gammagrafía de receptores de somatostatina (OctreoScan ${ }^{\circledR}$ ). Los estudios de estas imágenes combinadas pueden localizar el $80 \%$ de los tumores. Para evaluar la enfermedad metastási- 
ca tienen mayor utilidad y la TC y RM, siendo la RM más sensible que la TC en la identificación de metástasis hepáticas ${ }^{(2,8)}$. El muestreo venoso portal transhepático (THPVS, por sus iniciales en inglés), junto con la prueba de estimulación arterial selectiva con calcio, tienen una sensibilidad del $80 \%$ al $94 \%$ en la localización de los insulinomas ${ }^{(2,8)}$.

Después de la identificación del tumor, la cirugía está indicada para todas las neoplasias ubicadas. La elección del procedimiento dependerá de las características de la masa, su tamaño y su localización ${ }^{(7,11)}$.

El uso de la ecografía intraoperatoria (USIO) es útil para localizar lesiones intrapancreáticas, no palpables, y para determinar la proximidad de las lesiones del páncreas al conducto biliar. USIO realizadas durante una exploración abierta o laparoscópica pueden localizar un insulinoma en el 86\% de los $\operatorname{casos}^{(2)}$. La resección atípica, incluyendo la enucleación, o la pancreatectomía parcial, tienen la ventaja de preservar el parénquima pancreático tanto como sea posible, reduciendo el riesgo de insuficiencia exocrina/endocrina. Actualmente, la resección laparoscópica se ha realizado en insulinomas pequeños, los cuales se han considerado benignos, que se hallan localizados en el cuerpo o la cola del páncreas. El éxito se alcanza en un $70 \%$ a $100 \%$ de los casos $^{(11,17)}$. La resección radical se debe considerar para los pacientes en los que la lesión no es única o no está encapsulada, tumores mayores de $4 \mathrm{~cm}$ de diámetro o lesiones que se encuentran cerca del conducto pancreático principal. La linfadenectomía generalmente no se realiza $^{(2,7)}$

Aunque la tasa de curación después de la resección quirúrgica es muy alta, existe la posibilidad de complicaciones posoperatorias, especialmente con el desarrollo de fístula pancreática (15\% a $43 \%$ ), absceso intrabdominal (4\% a $6 \%$ ), y retraso del vaciamiento gástrico temporal (4\% a $8 \%)$ que se puede manejar con agentes farmacológicos ${ }^{(2,7)}$. En los casos en los que el tumor no pudo ser localizado durante la intervención, se realiza pancreatectomía distal ${ }^{(2,7)}$.

Para aquellos pacientes que no son candidatos quirúrgicos o que están a la espera del procedimiento se deben tomar medidas que busquen controlar los síntomas; entre ellas se incluyen: modificaciones en la dieta y agentes farmacológicos. La modificación de la dieta se basa en el consumo de comidas fraccionadas durante todo el día y a la mitad de la noche con el fin de evitar la hipoglucemia sintomática. El fármaco de elección inicial, y el más estudiado, es el diazóxido, con una dosis de inicio de 150 a $200 \mathrm{mg}$ administrados cada 8 o 12 horas (dosis máxima $400 \mathrm{mg} /$ día). El diazóxido ofrece control de los síntomas en aproximadamente el 50\% a 60\% de los pacientes. En la mitad de ellos se puede producir retención de sodio y presencia de edema, por lo que pueden requerir la adición de un diurético ${ }^{(1,2,3,4,11)}$.

Los análogos de la somatostatina como el octreótido y el lantreotide, se unen con alta afinidad a los receptores, logran- do disminuir los niveles de insulina en plasma y mejorando los síntomas en el $40 \%$ a $60 \%$ de los pacientes. Los bloqueadores de canales de calcio, como el verapamilo, y los bloqueadores beta como el propranolol, se han utilizado solos o en combinación con otros medicamentos para ayudar a controlar los síntomas en algunos pacientes. Al igual que los glucocorticoides y el glucagón, también se han administrado en unión al diazóxido, logrando en unos pocos, una leve mejoría ${ }^{(1,2,3,4,11)}$.

Los insulinomas malignos siguen siendo tumores muy raros. Para ser considerados malignos, se debe demostrar evidencia de infiltración local en el tejido circundante o debe haber compromiso de ganglios linfáticos o metástasis hepáticas. La incidencia de los insulinomas malignos oscila entre el $7 \%$ y el $10 \%$ de los casos, con una supervivencia de $29 \%$ a los 10 años $^{(9,18)}$.

El tamaño promedio de un insulinoma maligno al momento del diagnóstico varía desde 2,3 hasta $6,2 \mathrm{~cm}$, aunque no existe un umbral absoluto de tamaño que corresponda a malignidad. Referente a la ubicación intrapancreática, no existe un patrón claro, ciertos autores consideran que son más frecuentes en la localización caudal. Los principales sitios de metástasis o recurrencia son el hígado y los ganglios linfáticos regionales.

En pacientes con insulinomas malignos, la resección quirúrgica agresiva incluye resección pancreática extensa, resección hepática y trasplante de hígado, que se debe intentar siempre que sea posible para mejorar su supervivencia. Por otra parte, los tratamientos secundarios agresivos tales como quimioembolización o ablación por radiofrecuencia (ARF) pueden estar indicados para algunas metástasis hepáticas. En los pacientes con insulinomas malignos no resecables, se deben considerar estrategias para mejorar su calidad de vida, dentro de las que se incluyen la administración de octreótido junto con monitoreo continuo de la glucosa ${ }^{(9,18)}$.

Como regla general, los pacientes con insulinoma presentan mejoría mediante la resección quirúrgica del tumor. La necesidad de la localización preoperatoria de insulinoma y los métodos utilizados aún siguen siendo polémicos.

Anteriormente, la confirmación de la presencia de la tríada de Whipple significaba, por lo general, que un paciente debía ser llevado a cirugía; sin embargo, en la actualidad, la mayoría de autores están de acuerdo en que localizar el sitio del tumor antes del procedimiento es útil, ya que permite determinar no sólo si la enucleación del tumor o la resección pancreática son probables, sino también, si existe la posibilidad de extirpación a través de un abordaje laparoscópico ${ }^{(1,2,7,11,17,19,20)}$.

Luego de los hallazgos descritos en la presentación del caso, al igual que la correlación clínica y según la revisión de las investigaciones actuales, el diagnóstico de nuestro paciente corresponde a una hipoglucemia hiperinsulinémica secundaria a insulinoma. La localización del tumor por ultrasonografía endoscópica permitió planear una intervención quirúrgica con la que se logró la resección del insulinoma y la resolución, hasta el momento de su cuadro de hipoglucemia. 


\section{Referencias}

1. Aarti Mathur, Philip Gorden, Steven K. Libutti. Insulinoma. Surg Clin N Am 2009; 89. 1105-1121.

2. Takehiro Okabayashi, Yasuo Shima, Tatsuaki Sumiyoshi, Akihito Kozuki. Diagnosis and management of insulinoma. 2013; Volumen 19. 829-837.

3. Philip E. Cryer, Lloyd Axelrod, Ashley B. Grossman, Simon R. Heller. Evaluation and Management of Adult Hypoglycemic Disorders: An Endocrine Society Clinical Practice Guideline. J Clin Endocrinol Metab, 2009; 94(3):709728.

4. Jean-Marc Guettier, Phillip Gorden. Hypoglycemia. Clin Endocrinol Metab N Am, 2006; 35 753-766.

5. Fatima Louda, Asmaa Chadli, Siham Elaziz, Hassan Elghomari. Malignant insulinoma misdiagnosed and treated as epilepsy. Annales d' Endocrinologie, 2013; 74, 53-55.

6. Jérôme Aupy, Aurélien Benoilid, Maysa Sarhan, Constanza Dalvit. Misleading features of neuroimaging and electroencephalography: insulinoma misdiagnosed as temporal lobe epilepsy. Epileptic Disord, 2013; Vol. 15, No. 1.

7. Gerard J. Abood, Aileen Go, Deepak Malhotra, Margo Shoup. The Surgica and Systemic Management of Neuroendocrine Tumors of the Pancreas. Surg Clin N Am, 2009; 89 249-266.

8. Rachel B Lewis, Grant E. Lattin, Edina Paal. Pancreatic Endocrine Tumors: Radiologic-Clinicopathologic Correlation. RadioGraphics 2010; Volume 30 Number 6, 1445-1464.

9. Eric Baudin, Philippe Caron, Catherine Lombard-Bohas, Antoine Tabarin et al. Malignant insulinoma: Recommendations for characterisation and treatment. Annales d'Endocrinologie 2013; Vol 74, 523-533.

10. Wendy S. Rubinstein. Endocrine Cancer Predisposition Syndromes: Hereditary Paraganglioma, Multiple Endocrine Neoplasia Type 1, Multiple Endocrine Neoplasia Type 2, and Hereditary Thyroid Cancer. Hematol Oncol Clin N Am, 2010; 24, 907-937.
11. Katherine A. Morgan, David B. Adams. Solid Tumors of the Body and Tail of the Pancreas. Surg Clin N Am, 2010; 90, 287-307.

12. Robert T. Jensen, Guillaume Cadiot, Maria L. Brandi, Wouter W. de Herder ENETS Consensus Guidelines for the Management of Patients with Digestive Neuroendocrine Neoplasms: Functional Pancreatic EndocrineTumor Syndromes. Neuroendocrinology, 2012; 95:98-119.

13. Dizon AM, Kowalyk S, Hoogwerf BJ. Neuroglycopenic and other symptoms in patients with insulinomas. Am J Med, 1999; 106: 307-10.

14. Smith D, Defalla BA, Chadwick DW. The misdiagnosis of epilepsy and the management of refractory epilepsy in a specialist clinic. QJM, 1999; 92: 15 23.

15. Bazil CW, Pack A. Insulinoma presenting as seizure disorder. Neurology 2001; 56: 817-8

16. Piccillo GA, Musco A, Manfrini S, Mondati E, Guastella T. Two clinical cases of insulinoma misdiagnosed as psychiatric conditions. Acta Biomed, 2005 76: $118-22$.

17. David A. Kooby, Carrie K. Chu. Laparoscopic Management of Pancreatic Malignancies. Surg Clin N Am, 2010; 90 427- 446.

18. J. C. Ferrer-García, Iranzo González Cruz S, Navas-De Solís, Civera Andrés. Management of malignant insulinoma. Clin Transl Onco, 2013; 15:725-731.

19. Mehrabi A1, Fischer L, Hafezi M, A systematic review of localization, surgical treatment options, and outcome of insulinoma. Pancreas. 2014 Jul;43 (5):675-86.

20. AlJadir S (2015) Insulinoma: Literature's Review (Part 1). Endocrinol Metab Int J 2(3): 00025. DOI: 10.15406/emij.2015.02.00025

21. Vilchez MF, Ferreyra. Manifestaciones neuropsiquiátricas en insulinoma. Experiencia Médica. 2008. Vol 26-N 4: 122-126.

22. Valera Bestard, Soria Dorado. Manifestaciones psiquiátricas secundarias a las principales enfermedades endocrinologicas. Anales de medicina Interna. 2003. Vol 20. Número 4.206-212. 